\title{
Design of Signs Information Monitoring System for Man Overboard Based On BeiDou Satellite Navigation System
}

\author{
Shi-ai Ren ${ }^{1}$, Hong-wu Chen ${ }^{1}$, Hai-peng Zhang ${ }^{1}$, Zhu Liu $^{1} \&$ Shan-shan Wu ${ }^{1}$ \\ ${ }^{1}$ Department of College of Engineering Science and Technology, Shanghai Ocean University, \\ Shanghai, 201306, China
}

\begin{abstract}
Keywords: BeiDou orientation, real-time monitoring, signs information of human, sensors, wireless communication
\end{abstract}

\begin{abstract}
In order to meet the needs of the maritime rescue nowadays, a kind of real-time signs information monitoring system for man overboard based on BeiDou Satellite Navigation System is developed. A system has been proposed that it can monitor the location of the workers by adopting BeiDou Satellite Navigation System that is independently researched and developed by China, and measure information of human body signs by using the sensors, transmit data to the receiving terminal through the wireless communication module. Experiments has shown that the system has many advantages, such as low- cost, small volume, high precision and real-time performance strongly, stable and reliable, etc.
\end{abstract}

\section{Introduction}

As the development of economic, and the world's maritime trade growing, maritime activities become more frequent, and accidents at sea are happening with increasing frequency[1]. And as the complex environment of offshore marine, maritime accidents have occurred so sudden and catastrophic, however traditional life-saving equipment has been unable to meet existing usage. Satellite positioning rescue equipment emerged in recent years even though it can achieve positioning personnel, but it can not monitor the offshore workers healthy information[2]. It can more easily monitor the health of personnel through monitoring information, and more timely rescue the workers caused by the conduct of the sudden relief.

Human health is a reflection of the human body from external signs of information, and the signs of human information include temperature, blood pressure, pulse, respiratory rate, ECG (electrocardiogram) etc. The body temperature, blood pressure, pulse is to maintain the body's normal activities regardless of which abnormalities will cause serious or fatal condition[3]. For example, normal human body temperature is around $37^{\circ} \mathrm{C}$, and it can only survive about 15 minutes drowning in the water of $0^{\circ} \mathrm{C}$. When the water temperature is $15 \sim 20^{\circ} \mathrm{C}$, the drowning person can persist more than 10 hours[4]. In cold water, a huge loss of body heat will cause cramps, and seriously will case death. The special working environment at sea highlights the importance of monitoring offshore personnel information. Through monitoring the personnel information, not only can discover the abnormal situation happened, and by monitoring the location information, but also can pay close attention to the location of workers in time, and be able to provide important reference basis for the possibility of rescue activities.

\section{System Overall Structure Design}

Man overboard signs information monitoring system mainly includes two parts, the part is equipped by workers, one part is monitoring instruments on a ship. System adopts a simple Zig-Bee technology to form a network, and on the basis of the monitoring equipment in monitoring center, all equipped with facilities for the node, it will communicate through wireless with terminal equipment. Through forming a simple star-network, the system achieves the real-time monitoring and achieves personnel signs information real-time. System overall structure is shown in Fig.1. 


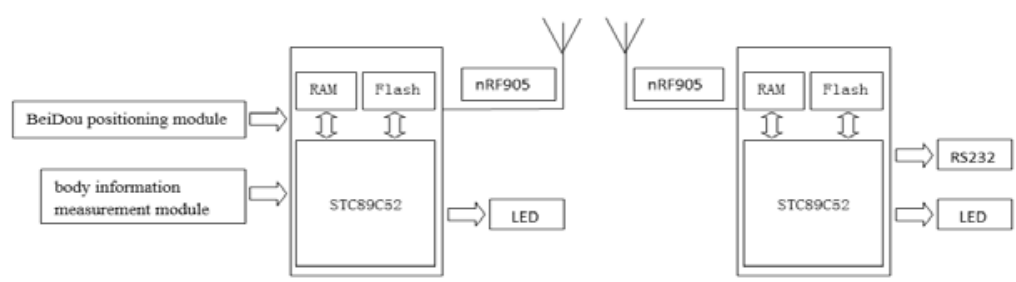

Fig.1: System overall structure

\section{The main functions of the monitoring instruments on the ship:}

i)to receive and display the current user's information, including the human body signs and personnel position information, real-time monitoring, to highlight abnormal situation, and to send out alarm information.

ii)the current request to join node can be judged, allow or prohibit to join, and you can delete the redundant nodes.

iii)receive data storage and backup.

\section{The main functions of the staff wear equipment:}

i)automatically search for control center, and put forward the application requirements.

ii)to collect signs information and location information, and send to monitoring terminal.

iii)automatic wake-up measuring terminal, real-time clock setting is used to measure the time interval, the complete time interval data collection.

iiii)monitor the usage of equipment power, and if power is insufficient, send out alarm information.

\section{The Design of The System Hardware Structure}

\section{Wireless communication module}

NRF905 is Nordic VLSI monolithic RF(Radio Frequency) transceiver chip start-up, the working voltage of $1.9 \mathrm{~V}$ to $3.6 \mathrm{~V}, 32$ pin QFN packages, working at $433 \mathrm{MHz}, 868 \mathrm{MHz}, 915 \mathrm{MHz}$ three ISM (Industrial Scientific Medical) channel, channel between the conversion time is less than 650 us[5]. The chip integrated frequency synthesizer, receiving demodulator, power amplifier, crystal oscillator and the modulator. It does not require additional acoustic wave filter, and power consumption is very low, receiving sensitivity of -100 DBM, built-in SPI interface communicating with microcontrollers, and configuration is very convenient.

In the open communication situation the distance of the chip is up to 300 meters. In Shock-Burst TM send mode, nRF905 automatically generate letter and CRC check code, and when sending process is completed, the pin data prepared notify the microprocessor data after launch. In receiving mode, AM of address matching and DR of data ready signals perform to MCU that data packets over a valid address have received[6]. Wireless transceiver circuit is shown in Fig.2.

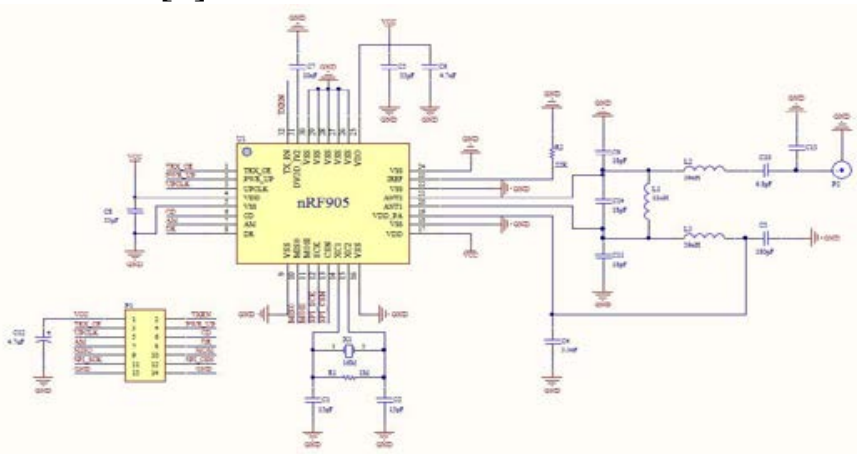

Fig.2: Wireless transceiver circuit design

According to the regulation of the international telecommunication union radio communications agency, this system wireless communication module choose the ISM (Industrial Scientific Medical) channels that are available, and this channel is mainly open to three main agencies including industry, science, medicine, belonging to the Free License without permission. On the $433 \mathrm{MHZ}$ channel it can realize point to multipoint communication, real-time monitor staff signs and 
positioning information, achieve real-time monitoring of offshore workers health.

\section{BeiDou Positioning Module}

BeiDou positioning module adopts a UM220 chip. The chip is a dual system of highperformance GNSS module, and has high positioning accuracy, fast speed measuring precision etc. The chip supports two frequency points, included BD2 B1 and GPS L1, packaging SMT solder with compact appearance. The chip adopts 3.3V voltage power supply, with input and output signal type of LVTTL level, through RS232 circuit transformation, realizing the serial communication with single-chip microcomputer or PC.

On the design of the hardware circuit, antenna signal using SMA (Sub-Miniature-A) radio interface, and using non $2.85 \mathrm{~V}$ active antenna, need to support power for the antenna separately. UM220 chip has three UART (Universal Asynchronous Receiver/Transmitter), outputs level type for LVTTL level, and baud rate ranges from 1200 bps to 230400 bps, communicating easily through the RS232 serial port with single-chip microcomputer and PC serial. And reset circuit adopts the $3.3 \mathrm{~V}$ power supply module increasing a power monitoring module MAX811, and connect it to the RST pin. BeiDou positioning circuit principle diagram is shown in Fig.3.

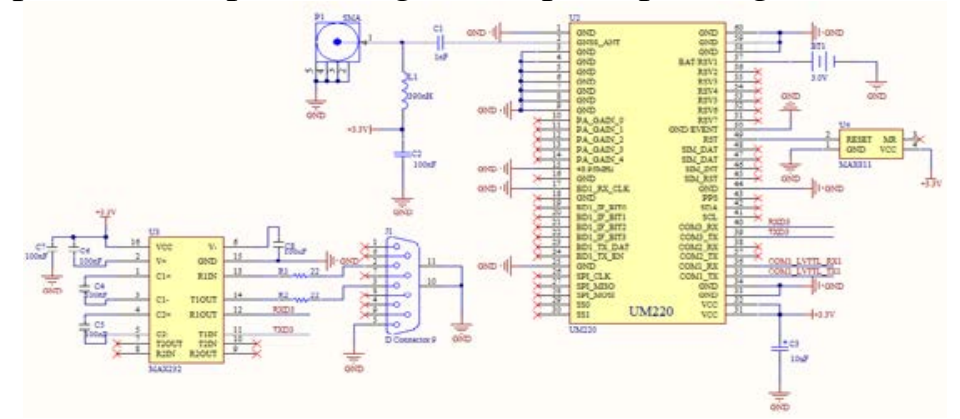

Fig.3: BeiDou positioning circuit principle diagram

\section{Signs Information Measurement Module}

Information signs is a healthy human body appearance. Due to working environment on the sea, signs information measurement and monitoring is particularly important. Blood pressure, pulse, temperature are three mainly signs information that maintain body to work normally, by monitoring the signs information of relevant staff can be more conducive to rescue on the sea. For example, multi-point temperature measurement adopts digital sensors DS18B20 measuring body temperature. The chip adopts the single bus, and can achieve multi-point temperature measurement, and measurement accuracy is $\pm 0.5^{\circ} \mathrm{C}$ from $-10^{\circ} \mathrm{C} \sim+85^{\circ} \mathrm{C}$. Multipoint temperature measurement circuit principle diagram as shown in Fig.4.

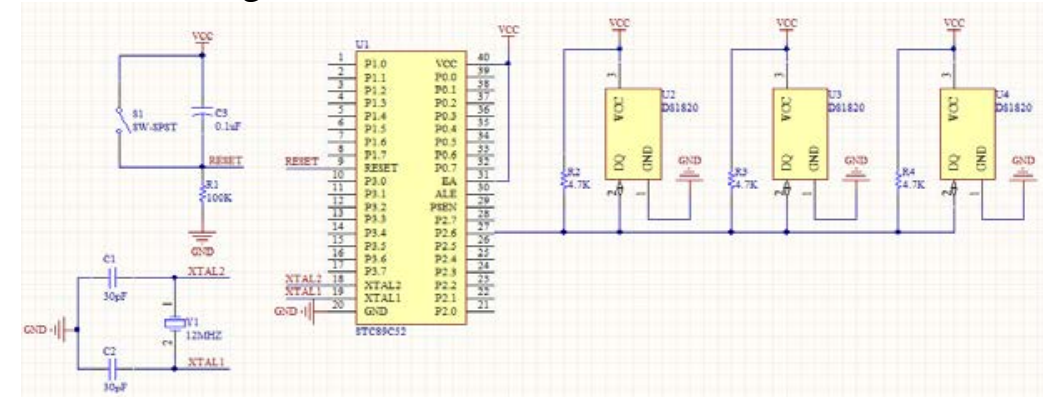

Fig.4: Multi-point temperature measurement circuit principle diagram

\section{The Structural Design of The System Software}

\section{The Design of The System Software}

The design of system software is an important component of the system, and the design needs to combine system hardware with software design that needs to implement the function of the corresponding. The system mainly realizes the signs for offshore operation personnel information and monitors location information, system program mainly using $C$ language to write, signs 
information measurement by personnel information module, positioning information calculating by BeiDou positioning UM220 module, and implementing short distance wireless communication with the monitoring center instruments on a ship through the nRF905 module. The monitoring center instruments on a ship receives personnel information that used by crews for real-time monitoring, and alarm for abnormal situation. The staff wearing equipment software working flow chart shown in Fig.5 and monitoring instruments on a ship software working flow chart shown in Fig.6.

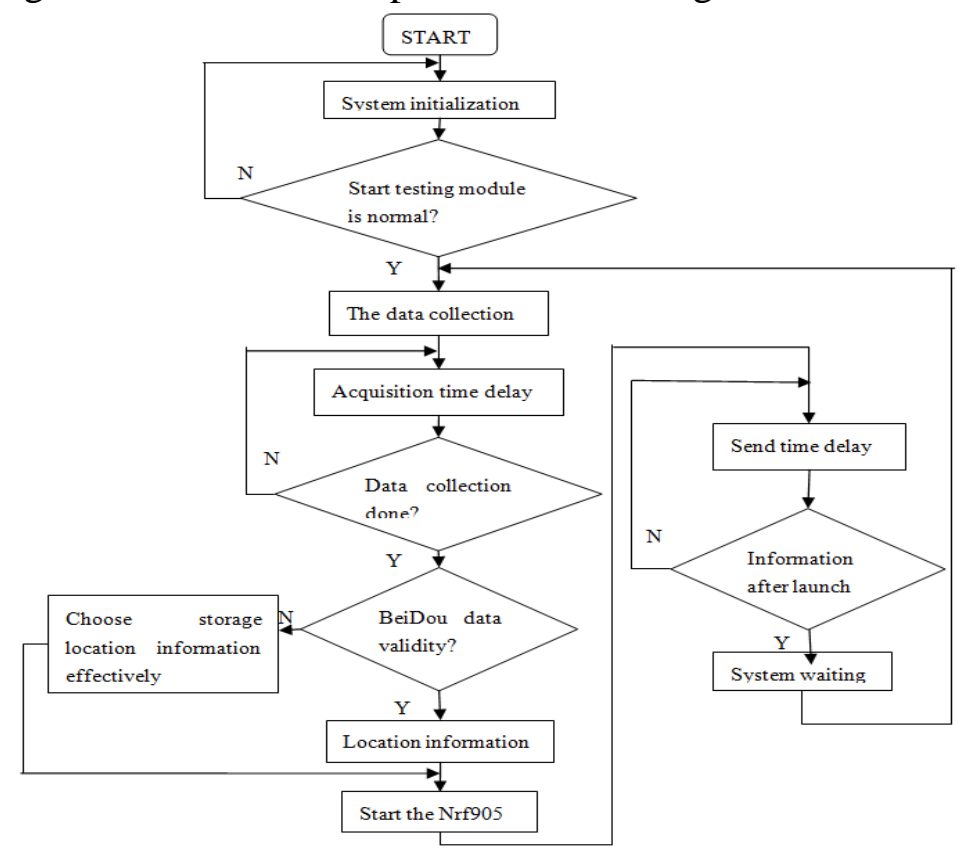

Fig.5: Staff wearing equipment software working flow chart

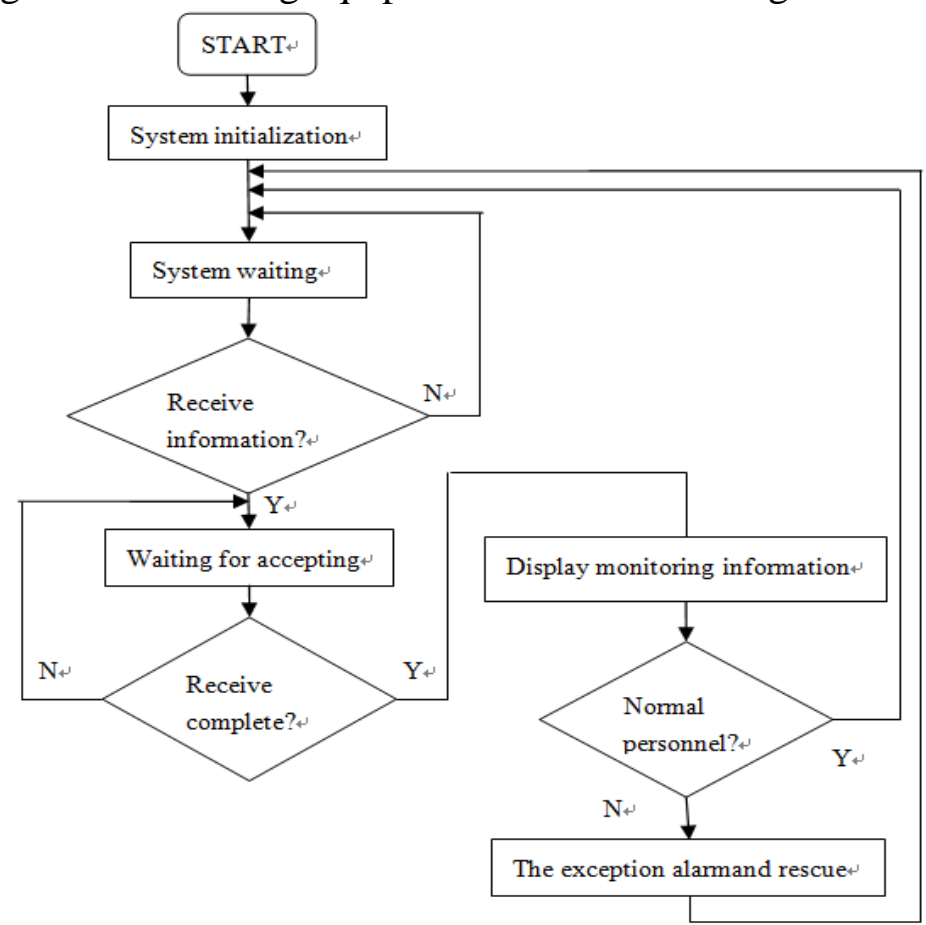

Fig.6: Monitoring instruments on a ship software working flow chart

\section{Wireless Data Transmission}

Through the nRF905 wireless modules, each signs information and location information will launch out at the same time. STC89C52 MCU I/O interface will connect module control port of RF mode, and SPI port will connect state output port, making the ordinary MCU interface simulate SPI interface. While send data, the controller will set TRX_CE and TX_EN pin to high level at the same time, and start frequency register, adding to the data and CRC check code. After the completion of sending data, DR will be set to high level, and TRX_CE is set to low, nRF905 data sending end and 
returning to standby mode. In receiving mode, TRX - CE is set to high and TX_EN is set to low at the same time, when the carrier detect pin (CD) is a high electricity at ordinary times, the controller will detect data, and at the same time address matching pin (AM) is set to high, accepting the correct packet, after accepting data complete data pin is set to high level, and TRX_CE is set to low. SPI interface is to read data validly, after completion of accepting the data DR pin and AM pin for low level.

\section{BeiDou Positioning Measurement}

The localization algorithm of UM220 BD2 B1 module adopts the least squares algorithm based on pseudo-range positioning, measuring staff position. Referring to the earth's rotation effect, users constantly request, and respond to the satellite signal. Through the two signal transmission delay, calculate the distance between the user and satellite, with the aid of the elevation of the location in which the users information, by using the theory of three ball intersection to measure users dilution of precision (DOP)[7]. The dilution of precision contains geometric dilution of precision GDOP, position dilution of precision PDOP, horizon dilution of precision HDOP, vertical dilution of precision VDOP, time dilution of precision TDOP, and GDOP value is less than 6, positioning error of the measuring data can meet the requirements of general orientation.

\section{The Experimental Result}

The staff wear equipment receives signs and position information, through the nRF905 wireless module communication with monitoring instruments on a ship for short distance. Monitoring instruments on a ship display personnel signs and position information, through the LED displaying alarm for the abnormal situation. For example, with "MYMBDGLL, 2953.2891, N, 2953.2891, E, $010941.000, \mathrm{~V}, \mathrm{~N} * 5 \mathrm{C} "$ message, the staff position is "29.532891 ${ }^{\circ} \mathrm{N}, 29.532891^{\circ} \mathrm{E} "$, the result shows as Fig.7.

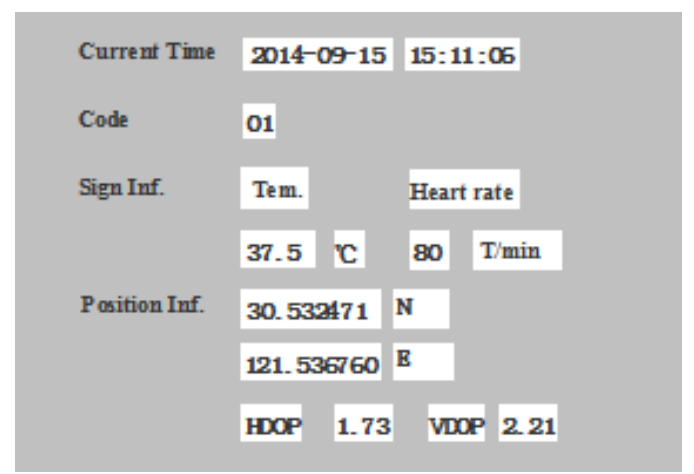

Fig.7: Monitoring center positioning result

\section{Conclusion}

This paper adopts by China BeiDou positioning system and radio frequency module, designing the maritime rescue system with features of practical and high reliability. When offshore personnel wears the device, it can realize to monitor information of offshore operation personnel by signs information sensor and UM220 positioning module, and real-time transmission to the center of the ship monitoring equipment, displaying the current personnel information of signs. When personnel risks, it can timely alarm to the center, taking effective rescue measures in time, for ensuring the safety of the workers. The experiments show that the system has reasonable design, convenient to use and accurate positioning features, for ensuring the safety of the offshore personnel on a small scale, and reducing operation risk. The system scheme is on the basis of the existing equipment research and development, not only conducive to the further development of Marine life-saving equipment research, and putting forward the signs information application in Marine life-saving equipment, to some extent, expanding the new vision of Marine life-saving equipment. 


\section{Acknowledgements}

Corresponding author: Hong-wu Chen. Department of College of Engineering Science and Technology, Shanghai Ocean University, Shanghai, 201306, China.

\section{Reference}

[1]Chen Bing-feng,Luan Ming-hao,Yang Chang-qing. Development of our national salvage and lifesaving equipments [J].World Shipping, 2005,28(4):14-15.

[2]WangDong-dong,ChenYong-jian,MaXiao-feng,WangWen-xu,LiuYang,Liu Kai. A GPS-andElectronic-Chart-Based Maritime Positioning [J]. Avionics Technology,2012,43(2):14-19.

[3]Pu Rui-rui. The research of vital signs monitoring system[D]. Xi AN: Xi AN Technology University,2013:31-37.

[4]Nie Shuang-shuang. All Kinds of self-help for victims at sea[J]. KEJISHIYE,2010,10(20):17-18.

[5]Ma Jin-xiang, He Yi-ming. Design on Wireless Transceiver System Based on nRF905 Module and AT89S MCU[J]. Communications Technology,2009,42(2):36-38.

[6]Qi Hong,Xu Zhi,Chen Chong,He Ming-hua. The design of short-distance wireless data transmission system based on Nrf905[J]. Journal of Fuzhou University(Natural Science), 2010,38(1):64-68.

[7]Zhao Yan-qing. Research of Location Algorithm and Analysis of GDOP of the COMPASS Satellite Navigation System[D]. Harbin:Harbin Engineering University,2013,3: 30-34. 